\title{
Study of Transferability of Rubus Microsatellite Markers to Hybrid Boysenberry
}

\author{
Jaihyunk Ryu ${ }^{3 \dagger}$, Woon Ji Kim ${ }^{1 \dagger}$, Juhyun $\mathrm{Im}^{1}$, Sang Hun Kim', Seung Cheol $\mathrm{Oh}^{2}$, Lan Cho ${ }^{2}$, Si-Yong Kang ${ }^{3}$, \\ Bo-Keun $\mathrm{Ha}^{1}$ * \\ ${ }^{1}$ Division of Plant Biotechnology, Chonnam National University, Gwangju 61186, Korea \\ ${ }^{2}$ Bioplus Co., Wanju 55310, Korea \\ ${ }^{3}$ Advanced Radiation Technology Institute, Korea Atomic Energy Research Institute, Jeongeup 56212, Korea
}

\begin{abstract}
Boysenberry, a Rubus hybrid between loganberry and a trailing blackberry, possesses distinctive polyphenol compounds, which have demonstrated positive biological effects on human health. Several new boysenberry genotypes have recently been developed from mutation breeding technology. In this study, a total of 103 SSR markers developed from expressed sequence tag (EST) and genomic libraries in blackberry and red raspberry were tested for cross-amplifications in 10 boysenberry genotypes. All primer pairs successfully produced amplification products, ranging from 1 to 4 loci per primer. Eleven polymorphic SSR markers (RH_MEa0007aB01, RH_MEa12cE03, RH_MEa14bF07, RH_MEa15aD04, RH_MEa13cF08, ERubLR_SQ01_N03, ERubLR_ SQ053_H01, ERubLR_SQ191_A05, RubfruitG7, Rubusr43a, and RiM019) were detected among boysenberry genotypes, while polymorphic loci were not detected in 92 markers. Polymorphism information content (PIC) and genetic diversity (GD) values ranged from 0.160 to 0.580 and from 180 to 0.640 , with average values of 0.359 and 0.407 , respectively, in the 11 polymorphic markers. According to a cluster analysis, all the mutant boysenberry genotypes can be classified into one category. Although the level of genetic diversity revealed by SSR markers in 10 boysenberry genotypes was low, these SSR markers will be useful for future genetic diversity, cultivar identification, QTL mapping, and gene cloning studies in boysenberry.
\end{abstract}

Keywords Boysenberry, SSR marker, Genetic diversity, Mutation, Gamma-ray

\section{INTRODUCTION}

Fruits of Rubus species are a rich source of anthocyanins and phenolics that provide a broad spectrum of biomedical functions in human health (Zafra-Stone et al. 2007). Boysenberry (Rubus ursinus Chamisso \& Schlenhtendal) is a Rubus hybrid between loganberry (Rubus loganobaccus Bailey) and a trailing blackberry (Rubus baileyanus Britt.) (Wood et al. 1999). Boysenberry fruits, which are usually consumed in fresh and processed products in human diet, contain a unique mixture of polyphenols including ellagitannins, short oligomeric proanthocyani- dins, anthocyanins, and ellagic acid (Furuuchi et al. 2011). Recent studies have indicated that the intake of polyphenols in boysenberry might offer positive effects such as reduction of oxidative stress (Barnett et al. 2007), prevention of liver injury (Igarashi et al. 2004), antihypertension (Furuuchi et al. 2012), and anti-obesity effects (Mineo et al. 2015). In addition, boysenberry had the highest level of free ellagic acid content among Rubus species (Wada and Ou 2002). The ellagic acid is well known as a potential anticarcinogenic compound against colon cancer, breast cancer, prostate cancer, and skin cancer (Zhang et al. 2014).

Received August 21, 2017; Revised October 13, 2017; Accepted October 16, 2017; Published December 1, 2017

*Corresponding author Bo-Keun Ha, bkha@jnu.ac.kr, Tel: +82-62-530-2055, Fax: +82-62-530-2059

${ }^{\dagger}$ These authors contributed equally. 
Generally, breeding progress in Rubus species has been limited by a lack of genetic variation for important agronomic traits in germplasms. Therefore, interspecific hybridization and mutation breeding constitute important breeding techniques in Rubus species. The inter-specific hybridization between red raspberry and blackberry have generated several new cultivars such as Loganberry, Nessberry, Phenomenal, and Brazos (Ipek et al. 2009). In addition, boysenberry is a "hybridberry." Mutation breeding has been used to improve specific agronomic traits such as bigger fruit sizes, early maturation, higher disease resistance, and higher anthocyanin content in fruits (Ryu et al. 2012; Ryu et al. 2016). Recently, a new blackberry cultivar, Maple, with high sugar content in fruit was developed via gamma-irradiation (Kim et al. 2013).

The narrow range of variation of morphological traits in Rubus species has thus far limited the accurate characterization of Rubus germplasm and cultivars. DNA markers could be a useful tool for cultivar identification and for the assessment of genetic diversity in breeding programs. In red raspberry, highly polymorphic and co-dominant simple sequence repeat (SSR) markers have been developed from genomic and cDNA libraries (Graham et al. 2002; Graham et al. 2004; Woodhead et al. 2008). Castillo et al. (2010) also developed SSR markers from genomic libraries of red raspberry and blackberry. These SSR markers could be transferable across Rubus species and have been used to differentiate between wild and cultivated Andean blackberry (Rubus glaucus) (Marulanda et al. 2012), to study genetic diversity in wild and cultivated black raspberry accessions (Dossett et al. 2012), and to construct a genetic map of tetraploid blackberry (Castro et al. 2013). However, there are no studies on the development or application of SSR markers in boysenberry. Only dominant AFLP markers have been applied to assess the genetic relationship between some blackberry cultivars and boysenberry (Ipek et al. 2009).

Recently, several boysenberry mutant lines showing high anthocyanin and ellagic acid contents have been developed via gamma-irradiation (Ryu et al. 2017). In this study, 103 SSR markers previously developed for other Rubus species were tested to determine their transferability in boysenberry and were used to assess the genetic diversity in boysenberry mutant genotypes.

\section{MATERIALS AND METHODS}

\section{Plant materials and DNA extraction}

Ten boysenberry genotypes were used in this study (Table 1). These genotypes were used to analyze fruit quality and antioxidant components in previous study (Ryu et al. 2017). The BS PI genotype, which is spiny, was introduced from Japan, and others included stabilized lines from advanced generations, all of which are thornless. The BS_Hybrid was developed from the cross between the thornless blackberry (R. fruticosus L. 'V3') and the BS_PI which was introduced from Japan (Shin et al. 2008). Six mutant genotypes (BSA-036 to BSA-144) were developed from 20 Gy gamma-ray treatments on explants of the hybrid boysenberry. Two mutant genotypes (BSB-032 and BSB-127) were developed from 40 Gy gamma-ray treat-

Table 1. List of 10 boysenberry genotypes evaluated with 103 SSR markers (Ryu et al. 2017).

\begin{tabular}{|c|c|c|c|c|}
\hline No. & Line & Origin & Treatment & Stem Spiny \\
\hline 1 & BS_PI & Boysenberry from Japan & & Spiny \\
\hline 2 & BS_Hybrid & Cross breeding & Blackberry(V3) $\times$ BS_PI & Thornless \\
\hline 3 & BSA-036 & Hybrid Boysenberry & Gamma-ray 20 Gy & Thornless \\
\hline 4 & BSA-065 & Hybrid Boysenberry & Gamma-ray 20 Gy & Thornless \\
\hline 5 & BSA-078 & Hybrid Boysenberry & Gamma-ray 20 Gy & Thornless \\
\hline 6 & BSA-101 & Hybrid Boysenberry & Gamma-ray 20 Gy & Thornless \\
\hline 7 & BSA-119 & Hybrid Boysenberry & Gamma-ray 20 Gy & Thornless \\
\hline 8 & BSA-144 & Hybrid Boysenberry & Gamma-ray 20 Gy & Thornless \\
\hline 9 & BSB-032 & Hybrid Boysenberry & Gamma-ray 40 Gy & Thornless \\
\hline 10 & BSB-127 & Hybrid Boysenberry & Gamma-ray 40 Gy & Thornless \\
\hline
\end{tabular}


ments on explants of the hybrid boysenberry. Freshly growing young leaves from each genotype were harvested for DNA samples. DNA was extracted using a DNeasy plant mini kit (Qiagen, Hilden, Germany) and quantified using a UV-Vis spectrophotometer (NanoDrop 2000, Thermo Scientific, DE, USA).

\section{SSR analysis}

A total of 103 SSR markers previously developed from expressed sequence tag (EST) and genomic libraries in blackberry (Rubus fruticosus L.) and red raspberry (Rubus idaeus L.) were used in this study. These primers are summarized in Table 2 and Supplementary Table S1. PCR amplification used a $10 \mu \mathrm{L}$ reaction mix containing $2 \mu \mathrm{L}$ of $40 \mathrm{ng}$ template DNA, $1.0 \times$ PCR buffer, $3.0 \mathrm{mM} \mathrm{MgCl}_{2}$, $100 \mu \mathrm{M}$ of each dNTP, $0.2 \mu \mathrm{M}$ each of forward and reverse primers, and 0.5 units of GoTaq DNA polymerase (Promega, Madison, USA). PCR cycling conditions were 30 second DNA denaturation step at $94^{\circ} \mathrm{C}$, a 30 second annealing step at $50^{\circ} \mathrm{C}$, and a 1 minute extension step at $72^{\circ} \mathrm{C}$ for 35 cycles on a TProfessional Basic thermocycler (Biometra, Göttingen, Germany). PCR amplifications were separated by capillary electrophoresis using a Fragment Analyzer system (Advanced Analytical Technologies, IA, USA) with dsDNA 900 Reagent Kit. After capillary electrophoresis separation, the SSR alleles were scored using PROSize 2.0 software (Advanced analytical Technologies, IA, USA).

\section{Data analysis}

Polymorphic validation and genetic diversity assessments, including polymorphism, gene diversity (GD), and polymorphism information content (PIC) of the alleles revealed by each primer pair were calculated from the genotype data from 10 boysenberry individuals using PowerMarker v3.25 software (Liu and Muse 2005). A phylogenetic dendrogram was constructed using the unweighted pair-group method with arithmetical algorithms averages (UPGMA) method by NTSYS PC 2.1 software (Exeter Software, NY, USA). Genetic distance matrix was calculated using Nei (1978)'s unbiased genetic distance measure.

\section{RESULTS}

\section{SSR marker selection}

A total of 103 primer pairs were tested against 10 boysenberry genotypes (Table 2). These SSR markers were selected from previous works. Among those, 53 SSR markers were developed from a blackberry and red raspberry genomic library, and 50 SSR markers were derived from a blackberry and red raspberry EST library (Graham et al. 2004; Lewers et al. 2008; Castillo et al. 2010; Castro et al. 2013; Woodhead et al. 2008). All primer pairs amplified a product in each of the 10 boysenberry genotypes that showed $100 \%$ transferability in boysenberry. Of these, only eleven SSR markers (10.7\%) revealed genetic differentiation among boysenberry genotypes, while polymorphic loci were not detected in 92 SSR primers (Table 2). Two out of 53 SSR markers (3.8\%) derived from the genomic library were found to be polymorphic for the 10 genotypes. However, 9 out of 50 SSR markers (18\%)

Table 2. Amplification profiles for 103 SSR markers tested in 10 boysenberry genotypes.

\begin{tabular}{|c|c|c|c|c|c|}
\hline Type of SSR markers & $\begin{array}{l}\text { Primer pairs } \\
\text { tested }\end{array}$ & $\begin{array}{l}\text { Amplification } \\
\text { success }(\%)\end{array}$ & $\begin{array}{c}\text { No. } \\
\text { polymorphic } \\
\text { markers }\end{array}$ & $\begin{array}{c}\text { Ratio of } \\
\text { polymorphic } \\
\text { markers }(\%)\end{array}$ & References \\
\hline Genomic SSR of blackberry & 4 & 100 & 0 & 0 & Castro et al. (2013) \\
\hline $\begin{array}{l}\text { Genomic SSR of blackberry } \\
\text { and red raspberry }\end{array}$ & 10 & 100 & 1 & 10 & Castillo et al. (2010) \\
\hline Genomic SSR of red raspberry & 39 & 100 & 1 & 2.6 & Graham et al. (2004) \\
\hline EST SSR of blackberry & 19 & 100 & 5 & 26.3 & Lewers et al. (2008) \\
\hline EST SSR of red raspberry & 23 & 100 & 3 & 13.0 & Woodhead et al. (2008) \\
\hline EST SSR of red raspberry & 8 & 100 & 1 & 12.5 & Graham et al. (2004) \\
\hline Total (Mean) & 103 & $(100)$ & 11 & $(10.7)$ & \\
\hline
\end{tabular}


derived from the EST library were polymorphic. Therefore, the polymorphic rate of EST-SSR markers was approximately 4.7 times higher than that of genomic-SSR markers in this study. In addition, the blackberry-derived EST-SST markers showed the highest polymorphic rates $(26.3 \%)$ among boysenberry genotypes.

The number of loci, number of polymorphic loci, and polymorphic rate of boysenberry genotypes are presented in Supplementary Table S1. Polymorphism analysis detected 123 loci generated from all 103 SSR primer set, of which 27 loci were polymorphic, representing $21.9 \%$ of the total generated loci. The total number of loci per primer ranged from 1 to 4. Eight SSR primers (ERubLR_SQ01_ N03, ERubLR_SQ191_A05, RH_MEa0007aB01, RiM019, Rubusr43a, RH_MEa12cE03, RH_MEa15aD04, and RH_ MEa13cF08) gave the highest percentage of polymorphic loci $(100 \%)$.

The observed number of alleles (Na), effective number of alleles (Ne), gene diversity (GD) and PIC of boysenberry genotypes were analyzed with 11 polymorphic SSR markers (Table 3). The mean Na value was 1.909 and ranged from 1.5 to 2.0. The mean Ne value was 1.421 and

Table 3. Characteristics of 11 SSR markers showing polymorphisms in 10 boysenberry genotypes.

\begin{tabular}{cllcccc}
\hline \hline No. & \multicolumn{1}{c}{ Marker name } & \multicolumn{1}{c}{ Type of SSR } & $\mathrm{Na}^{\mathrm{z})}$ & $\mathrm{Ne}^{\mathrm{y})}$ & $\mathrm{GD}^{\mathrm{x})}$ & $\mathrm{PIC}^{\mathrm{w})}$ \\
\hline 1 & RH_MEa0007aB01 & EST SSR of blackberry & 2.000 & 1.458 & 0.640 & 0.580 \\
2 & RH_MEa12cE03 & EST SSR of blackberry & 2.000 & 1.220 & 0.340 & 0.310 \\
3 & RH_MEa14bF07 & EST SSR of blackberry & 2.000 & 1.584 & 0.640 & 0.580 \\
4 & RH_MEa15aD04 & EST SSR of blackberry & 2.000 & 1.395 & 0.580 & 0.540 \\
5 & RH_MEa13cF08 & EST SSR of blackberry & 2.000 & 1.490 & 0.180 & 0.160 \\
6 & ERubLR_SQ01_N03 & EST SSR of red raspberry & 2.000 & 1.220 & 0.180 & 0.164 \\
7 & ERubLR_SQ053_H01 & EST SSR of red raspberry & 1.500 & 1.500 & 0.420 & 0.330 \\
8 & ERubLR_SQ191_A05 & EST SSR of red raspberry & 2.000 & 1.345 & 0.620 & 0.540 \\
9 & RubfruitG7 & EST SSR of red raspberry & 2.000 & 1.490 & 0.180 & 0.164 \\
10 & Rubusr43a & Genomic SSR of red raspberry & 2.000 & 1.571 & 0.380 & 0.310 \\
11 & RiM019 & Genomic SSR of blackberry & 1.500 & 1.362 & 0.320 & 0.270 \\
& & and red raspberry & & & & \\
& & Mean & 1.909 & 1.421 & 0.407 & 0.359 \\
\hline
\end{tabular}

${ }^{\text {z) }} \mathrm{Na}$ : Observed number of alleles, ${ }^{\mathrm{y})} \mathrm{Ne}$ : Effective number of alleles, ${ }^{\mathrm{x}} \mathrm{GD}$ : Gene diversity, ${ }^{\mathrm{w})} \mathrm{PIC}$ : Polymorphism information content.

Table 4. Putative function of EST-SSR markers showing polymorphisms in 10 boysenberry genotypes.

\begin{tabular}{|c|c|c|c|c|}
\hline No. & Marker name & $\begin{array}{l}\text { GenBank } \\
\text { accession no. }\end{array}$ & Homology & E-value \\
\hline 1 & RH_MEa0007aB01 & FF683655 & Fragaria vesca subsp. vesca E3 ubiquitin-protein ligase SIS3 & $1 \mathrm{e}-155$ \\
\hline 2 & RH_MEa12cE03 & FF684289 & Fragaria vesca subsp. vesca uncharacterized LOC101302468 & $1 \mathrm{e}-119$ \\
\hline 3 & RH_MEa14bF07 & FF684762 & $\begin{array}{l}\text { Fragaria vesca subsp. vesca chlorophyll a-b binding protein of } \\
\text { LHCII type 1-like }\end{array}$ & 0.0 \\
\hline 4 & RH_MEa15aD04 & FF684949 & Fragaria $x$ ananassa zeta-carotene desaturase protein (zds) & 0.0 \\
\hline 5 & RH_MEa13cF08 & FF684532 & $\begin{array}{l}\text { Fragaria vesca subsp. vesca ranBP2-type zinc finger protein } \\
\text { At1g67325 }\end{array}$ & $2 \mathrm{e}-31$ \\
\hline 6 & ERubLR_SQ01_N03 & EX567290 & $\begin{array}{l}\text { Fragaria vesca subsp. vesca major allergen Pru av 1-like } \\
\text { (LOC101298594) }\end{array}$ & $3 e-121$ \\
\hline 7 & ERubLR_SQ053_H01 & EX567274 & $\begin{array}{l}\text { Rosa } x \text { bourboniana xyloglucan } \\
\text { endotransglucosylase/hydrolase } 8 \text { (XTH8) }\end{array}$ & $1 \mathrm{e}-78$ \\
\hline 8 & ERubLR SQ191 A05 & EX567282 & Prunus mume RING-H2 finger protein ATL20-like & $6 e-40$ \\
\hline
\end{tabular}


ranged from 1.220 (ERubLR_SQ01_N03) to 1.584 (RH_ MEa14bF07). The GD for 11 polymorphic SSR markers ranged from 0.180 to 0.640 , with a mean of 0.407 . The PIC of the 11 polymorphic SSR markers ranged from 0.160 to 0.580 , with an average of 0.359 . The maximum values of GD (0.640) and PIC (0.580) occurred with the RH MEa0007aB01 and RH_MEa14bF07 primers that were derived from the EST library for blackberry.

EST-SSR markers associated with expressed genes are useful to study functional diversity in populations. The putative function of 8 EST-SSR markers showing high PIC values were determined by using BLAST against the non-redundant GenBank database at the National Center for Biotechnology Information (NCBI) (Table 4). The 8 EST sequences showed significant similarities to known genes including E3 ubiquitin-protein ligase, chlorophyll a-b binding protein, zeta-carotene desaturase protein, allergen Pru av1-like, xyloglucan endotransglucosylase/ hydrolase 8, and RING-H2 finger protein. The most homologies were from Fragaria vesca.

\section{Genetic distance and cluster analysis}

Genetic distance matrix (GDM) obtained from 27 polymorphic loci were shown in Table 5. The scale used for the genetic distance runs from 0 (meaning no genetic difference) to 0.898 with an average of 0.268 . BSA-119 (No. 7), BSA-114 (No. 8), and BSB-127 (No. 10) exhibited no genetic difference among genotypes. Original cultivar (Hybrid boysenberry) and BSA-036 were the most distantly related.
Based on the simple-matching coefficient between boysenberry genotype, a cluster analysis was carried out and a dendrogram was generated with 27 polymorphic loci via the average linkage method for genetic relationships (Fig. 1). According to cluster analysis of the SSR markers, boysenberry genotypes could be divided into a gamma-raytreated group (BSA-036, BSA-065, BSA-078, BSB-032, BSA-101, BSB-127, BSA-119 and BSA-144) and a nontreatment group (boysenberry and hybrid boysenberry), divided at the genetic distance of 0.485 . However, a gamma-ray-treated group was not classified according to the dosage of gamma rays in the dendrogram.

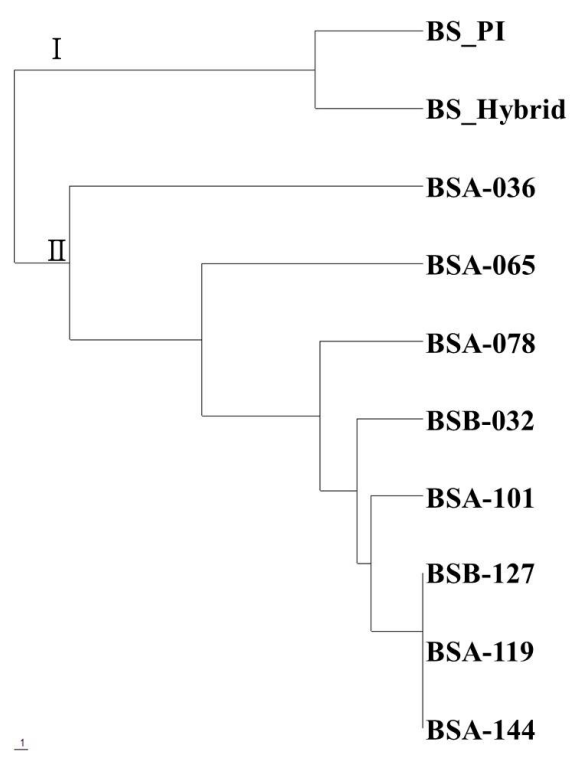

Fig. 1. Phylogenetic tree based on boysenberry genotypes of 11 polymorphic SSR markers.

Table 5. Matrix of Nei (1978)'s genetic distances between boysenberry genotype.

\begin{tabular}{ccccccccccc}
\hline \hline Line $\mathrm{No}^{\mathrm{z}}$ & 1 & 2 & 3 & 4 & 5 & 6 & 7 & 8 & 9 & 10 \\
\hline 1 & 0.000 & & & & & & & & & \\
2 & 0.160 & 0.000 & & & & & & & & \\
3 & 0.898 & 0.898 & 0.000 & & & & & & & \\
4 & 0.463 & 0.463 & 0.588 & 0.000 & & & & & & \\
5 & 0.406 & 0.406 & 0.523 & 0.205 & 0.000 & & & & & \\
6 & 0.588 & 0.588 & 0.588 & 0.251 & 0.205 & 0.000 & & & & \\
7 & 0.588 & 0.588 & 0.463 & 0.351 & 0.118 & 0.077 & 0.000 & & & \\
8 & 0.588 & 0.588 & 0.463 & 0.351 & 0.118 & 0.077 & 0.000 & 0.000 & & \\
9 & 0.731 & 0.731 & 0.588 & 0.463 & 0.205 & 0.160 & 0.077 & 0.077 & 0.000 & \\
10 & 0.588 & 0.588 & 0.463 & 0.351 & 0.118 & 0.077 & 0.000 & 0.000 & 0.077 & 0.000 \\
\hline
\end{tabular}

${ }^{2)}$ Line No.: Line number is listed in Table 1 . 


\section{DISCUSSION}

In berry crops, mutation breeding has already been used to introduce many useful traits affecting fruit quality, phytochemicals, disease resistance, resistance to low temperature, and the presence or absence of thorns (Basaran and Kepenek 2011; Ryu et al. 2016). In this study, the 'BS_Hybrid' genotype, obtained from crosses between the thornless blackberry (R. fruticosus L. 'V3') and boysenberry, has unfavorable fruit characteristics such as malformed fruit, small size, and lower sugar content. However, the mutant genotypes, obtained from gamma-ray mutations with 'BS_Hybrid', showed improved agronomic characteristics such as higher fruit yields, sugar contents, and antioxidant components when compared with the original parent (Ryu et al. 2017). These mutant genotypes can be directly released as new cultivars.

Molecular markers were useful tools for the evaluation of genetic diversity, marker-assisted breeding, DNA fingerprinting, and cultivar identification in crops. However, there are many challenges in developing molecular markers in Rubus species for which genomic information is not available. Therefore, previous studies have analyzed the genetic diversity of boysenberry and blackberry using AFLP markers (Ipek et al. 2009; Ryu et al. 2014). While no sequence information is needed, the AFLP marker system is not as easy to perform as SSR markers, which show highly reproducible, co-dominant, multi-allelic, PCRbased, and medium throughput to assay (Dossett et al. 2012; Stafne et al. 2005). In particular, SSR markers developed for one plant species can be transferred between related species (Varshney et al. 2005). The high level of transferability of SSR markers could reduce the costs of marker development in boysenberry. In this study, a total of 103 SSR markers previously developed from EST and genomic libraries in blackberry and red raspberry showed $100 \%$ transferability to 10 boysenberry genotypes. The high level of transferability of SSR markers among three Rubus species means that they have a close genetic relationship with each other (Ipek et al. 2009; Lee et al. 2015). Similarly, high rates of transferability of SSR markers were reported in pine $(94.2 \%$ between Pinus taeda and Pinus radiata), buckwheat (97.1\% between Fagopyrum esculen- tum and Fagopyrum homotropicum), and onion (93.5\% between Allium fistulosum and Allium altaicum) (Araki et al. 2010; Chagné et al. 2004; Ma et al. 2009).

Screening for polymorphism of SSR markers rendered the 11 polymorphic markers $(10.7 \%)$ in boysenberry genotypes. This rate is low compared to the $18 \%$ to $30 \%$ polymorphisms between the two blackberry genotypes using SSR markers derived from Rubus and Fragaria L. (Castro et al. 2013; Stafne et al. 2005). The lower rate of polymorphism may be the result of the eight boysenberry genotypes developed from one BS_Hybrid through gammaray mutation techniques, as well as BS_Hybrid derived from the cross with the BS_PI. However, the blackberryderived EST-SSR markers showed the $26.3 \%$ polymorphisms that had similar rates to the previous two studies of Castro et al. (2013) and Stafne et al. (2005).

The EST-SSR markers representing transcribed genes can be used for assaying functional diversity in accessions as well as yielding a higher rate of polymorphism (Varshney et al. 2005). Nine polymorphic SSR markers were developed from a blackberry leaf and raspberry root and fruit EST library (Graham et al. 2004; Lewers et al. 2008; Woodhead et al. 2008). The putative functions of two EST-SSR markers (RH_MEa0007aB01 and RH_ MEa14bF07) showing the highest PIC value $(0.580)$ were similar with Fragaria vesca subsp. vesca E3 ubiquitinprotein ligase and chlorophyll a-b binding protein, respectively (Table 4). Interestingly, the putative function of RH_MEa15aD04 marker was similar to zeta-carotene desaturase protein essential for carotenoid biosynthesis (Nisar et al. 2015). Avendaño-Vázquez et al. (2014) reported that the mutation of zeta-carotene desaturase caused severe alterations in leaf morphology. However, any abnormal leaf morphology was not detected within our boysenberry genotypes. In addition, the ERubLR_SQ01_ N03 marker showing the putative function of major allergen Pru av1 (Prunus avium) can be used as a potential diagnostic marker for severe allergic reactions to boysenberry genotypes (Scheurer et al. 1997).

In the clustering analysis, the eight gamma-ray mutagentreated genotypes and the original genotypes (BS_PI and BS_Hybrid) were divided into independent groups. Ryu et al. (2014) reported that blackberry mutants could be 
divided into gamma-ray and MNU-treated groups and crossbred (R. fruticosus $\times R$. parvifolius) lines by AFLP markers. Also, individuals of Cymbidium spp. were divided into control and electron beam treatment groups using both ISSR and RAPD markers (Ryu et al. 2013). These results suggest that radiation could induce significant genetic variability in boysenberry genotypes. The GDM among boysenberry genotypes ranged from 0 to 0.898 with an average 0.268 , which was similar to 11 cultivars of boysenberry and blackberry spanning from 0 to 0.85 by AFLP analysis (Ipek et al. 2009).

Analysis of genetic diversity and relationships may be helpful for QTL mapping and for gene cloning studies. This study also provides 11 polymorphic SSR markers among the boysenberry genotypes that would be useful for identification of cultivars. Therefore, these results may contribute to the selection of parental lines for both traditional and mutational breeding of boysenberry.

\section{ACKNOWLEDGEMENTS}

This study was supported by the Korea Institute of Planning and Evaluation for Technology in Food, Agriculture, Forestry and Fisheries (IPET) through the AgriBio Industry Technology Development Program, funded by the Ministry of Agriculture, Food and Rural Affairs (MAFRA), Republic of Korea (315025-03-1-HD060).

\section{REFERENCES}

Araki N, Masuzaki S-I, Tsukazaki H, Yaguchi S, Wako T, Tashiro Y, et al. 2010. Development of microsatellite markers in cultivated and wild species of sections Cepa and Phyllodolon in Allium. Euphytica 173: 321-328.

Avendaño-Vázquez A-O, Cordoba E, Llamas E, San Román C, Nisar N, De la Torre S, et al. 2014. An uncharacterized apocarotenoid-derived signal generated in $\zeta$-carotene desaturase mutants regulates leaf development and the expression of chloroplast and nuclear genes in Arabidopsis. Plant Cell 26: 2524-2537.

Barnett LE, Broomfield AM, Hendriks WH, Hunt MB, McGhie TK. 2007. The in vivo antioxidant action and the reduction of oxidative stress by boysenberry extract is dependent on base diet constituents in rats. J. Med. Food 10: 281-289.

Basaran P, Kepenek K. 2011. Fruit quality attributes of blackberry (Rubus sanctus) mutants obtained by ${ }^{60} \mathrm{Co}$ gamma irradiation. Biotechnol. Bioprocess Eng. 16: 587-592.

Castillo NRF, Reed BM, Graham J, Fernández-Fernández F, Bassil NV. 2010. Microsatellite markers for raspberry and blackberry. J. Am. Soc. Hortic. Sci. 135: 271-278.

Castro P, Stafne ET, Clark JR, Lewers KS. 2013. Genetic map of the primocane-fruiting and thornless traits of tetraploid blackberry. Theor. Appl. Genet. 126: 2521-2532.

Chagné D, Chaumeil P, Ramboer A, Collada C, Guevara A, Cervera M, et al. 2004. Cross-species transferability and mapping of genomic and cDNA SSRs in pines. Theor. Appl. Genet. 109: 1204-1214.

Dossett M, Bassil NV, Lewers KS, Finn CE. 2012. Genetic diversity in wild and cultivated black raspberry (Rubus occidentalis L.) evaluated by simple sequence repeat markers. Genet. Resour. Crop Evol. 59: 1849-1865.

Furuuchi R, Sakai H, Hirokawa N, Watanabe Y, Yokoyama T, Hirayama M. 2012. Antihypertensive effect of boysenberry seed polyphenols on spontaneously hypertensive rats and identification of orally absorbable proanthocyanidins with vasorelaxant activity. Biosci. Biotechnol. Biochem. 76: 1694-1701.

Furuuchi R, Yokoyama T, Watanabe Y, Hirayama M. 2011. Identification and quantification of short oligomeric proanthocyanidins and other polyphenols in boysenberry seeds and juice. J. Agric. Food Chem. 59: 3738-3746.

Graham J, Smith K, MacKenzie K, Jorgenson L, Hackett C, Powell W. 2004. The construction of a genetic linkage map of red raspberry (Rubus idaeus subsp. idaeus) based on AFLPs, genomic-SSR and EST-SSR markers. Theor. Appl. Genet. 109: 740-749.

Graham J, Smith K, Woodhead M, Russell J. 2002. Development and use of simple sequence repeat SSR markers in Rubus species. Mol. Ecol. Notes 2: 250-252.

Igarashi K, Sugimoto E, Hatakeyama A, Molyneux J, Kubomura K. 2004. Preventive effects of dietary boysenberry anthocyanins on galactosamine-induced liver injury in rats. Biofactors 21: 259-261.

Ipek A, Barut E, Gulen H, Ipek M. 2009. Genetic diversity among some blackberry cultivars and their relationship 
with Boysenberry assessed by AFLP Markers. Afr. J. Biotechnol. 8: 4830-4834.

Kim E-Y, Yoon TY, Jo H-J, Yu YR. 2013. New blackberry cultivar Maple, p. 1-9. In: KIPO (ed.). Korean Stevia Co., Korea.

Lee G-A, Song J, Choi H-R, Chung J-W, Jeon Y-A, Lee J-R, et al. 2015. Novel microsatellite markers acquired from Rubus coreanus Miq. and cross-amplification in other Rubus species. Molecules 20: 6432.

Lewers KS, Saski CA, Cuthbertson BJ, Henry DC, Staton ME, Main DS, et al. 2008. A blackberry (Rubus L.) expressed sequence tag library for the development of simple sequence repeat markers. BMC Plant Biol. 8: 1-8.

Liu K, Muse SV. 2005. PowerMarker: an integrated analysis environment for genetic marker analysis. Bioinformatics 21: 2128-2129.

Ma K-H, Kim N-S, Lee G-A, Lee S-Y, Lee JK, Yi JY, et al. 2009. Development of SSR markers for studies of diversity in the genus Fagopyrum. Theor. Appl. Genet. 119: 1247-1254.

Marulanda M, Lopez AM, Uribe M. 2012. Molecular characterization of the Andean blackberry, Rubus glaucus, using SSR markers. Genet. Mol. Res. 11: 322-331.

Mineo S, Noguchi A, Nagakura Y, Kobori K, Ohta T, Sakaguchi E, et al. 2015. Boysenberry polyphenols suppressed elevation of plasma triglyceride levels in rats. J. Nutr. Sci. Vitaminol. 61: 306-312.

Nei M. 1978. Estimation of average heterozygosity and genetic distance from a small number of individuals Genetics 89: 583-590.

Nisar N, Li L, Lu S, Khin NC, Pogson BJ. 2015. Carotenoid Metabolism in Plants. Mol. Plant 8: 68-82.

Ryu J, Ha B-K, Kim Ds, Kim J-B, Kim SH, Ahn J-W, et al. 2014. Genetic diversity and relationship assessment based on AFLP analysis in blackberry (Rubus fructicosus L.) mutant lines. Plant Breed. Biotech. 2: 386-395.

Ryu J, Kim DS, Ha B-K, Kim J-B, Kim SH, Jeong IY, et al. 2012. Growth characteristics and morphological variation analysis of mutant lines derived from gamma-ray and chemical mutagen treatments in Rubus fructicosus L. Journal of Radiation Industry 6: 257-265.

Ryu J, Kwon S-J, Jo YD, Choi H-I, Kang K-Y, Nam BM, et al. 2017. Fruit quality and chemical contents of hybrid boysenberry (Rubus ursinus) lines developed by hybridization and gamma irradiation. Plant Breed. Biotech. 5:
228-236.

Ryu J, Kwon S-J, Jo YD, Jin CH, Nam BM, Lee SY, et al. 2016. Comparison of phytochemicals and antioxidant activity in blackberry (Rubus fruticosus L.) fruits of mutant lines at the different harvest time. Plant Breed. Biotech. 4: 242-251.

Ryu J, So H, Bae S, Kang H, Lee B, Kang S, et al. 2013. Genetic diversity of in vitro cultured Cymbidium spp. irradiated with electron beam. Korean J. Breed. Sci. 45: 8-18.

Scheurer S, Metzner K, Haustein D, Vieths S. 1997. Molecular cloning, expression and characterization of Pru a 1, the major cherry allergen. Molecular Immunology 34: 619-629.

Shin J-S, Lee J-C, Sim O-K, Yoon T-Y, Cho H-J, Kim E-Y. 2008. Plant regeneration via multiple shoot formation from sucker explant of hybrid blackberry (Rubus fruticosus L. $\times$ R. parvifolius L.). Korean J. Plant Res. 21: 111-116. Stafne ET, Clark JR, Weber CA, Graham J, Lewers KS. 2005. Simple sequence repeat (SSR) markers for genetic mapping of raspberry and blackberry. J. Am. Soc. Hortic. Sci. 130: 722-728.

Varshney RK, Graner A, Sorrells ME. 2005. Genic microsatellite markers in plants: features and applications. Trends Biotechn. 23: 48-55.

Wada L, Ou B. 2002. Antioxidant activity and phenolic content of oregon caneberries. J. Agric. Food Chem. 50: 3495-3500.

Wood GA, Andersen MT, Forster RLS, Braithwaite M, Hall HK. 1999. History of Boysenberry and Youngberry in New Zealand in relation to their problems with Boysenberry decline, the association of a fungal pathogen, and possibly a phytoplasma, with this disease. N. Z. J. Crop Hortic. Sci. 27: 281-295.

Woodhead M, McCallum S, Smith K, Cardle L, Mazzitelli L, Graham J. 2008. Identification, characterisation and mapping of simple sequence repeat (SSR) markers from raspberry root and bud ESTs. Mol. Breed. 22: 555-563.

Zafra-Stone S, Yasmin T, Bagchi M, Chatterjee A, Vinson JA, Bagchi D. 2007. Berry anthocyanins as novel antioxidants in human health and disease prevention. Mol. Nutr. Food. Res. 51: 675-683.

Zhang H-M, Zhao L, Li H, Xu H, Chen W-W, Tao L. 2014. Research progress on the anticarcinogenic actions and mechanisms of ellagic acid. Cancer Biol. Med. 11: 92-100. 